\title{
The k-distance coloring and k-distance edge coloring on double graph of paths
}

\author{
Susu Jiao ${ }^{1, a}$, Shuangliang Tian ${ }^{2, b, *}$, Xiahong Cai, Huan Yang \\ 1,2,3,4 College of Mathematics and Computer ScienceNorthwest Minzu University Lanzhou China \\ a Email:jiaosu0806@163.com, bEmail:sl_tian@163.com \\ ${ }^{*}$ Corresponding author
}

Keywords: path, $k$-distance colorings, $k$-distance edge colorings, double graphs, semistrong product.

Abstract. In this paper, we study the $k$-distance colorings and $k$-distance edge colorings of path double graphs which are based on the semistrong product. We shows that two formulas about $k$-distance and $k$-distance edge chromatic number of path double graphs vary with $k$.

\section{Introduction}

The coloring problem of the graphs is essentially the smallest classification problem of the graph elements. The normal coloring edge coloring of the graph classify to the sets of vertex or edge, each of which is an independent set. The new concepts of distance coloring and distance edge coloring are based on traditional coloring concepts proposed by adding restrictions. A $k$-distance coloring of a graph $G$ is the distance of two distinct vertices less than or equal to $k$ must be assigned different colors. The minimum number of colors required for a $k$-distance coloring of $G$ denoted by $\chi_{k}(G)$.

In [1-4], F. Kramer and H. Kramer have introduced the new concept of $k$-distance coloring, Kramer defines a $k$-power graph of $G$ to illustrate that $k$-distance chromatic number equals the number of $k$-power graph of $G$. Besides many results have been demonstrated: for planar graphs $G, \chi_{k}(G)=k+1$, only if the following conditions are satisfied: (1) The vertices of $G$ satisfies $|V|=k+1$; (2) The path $G$ is the way of length greater than $k$.(3) The length of a cycle $G$ is an integer multiple of $k+1$. Fertin et al. in [5] have studied $k$-distance coloring problem of 2 dimensional grid (or the Cartesian product of two paths), the following conclusions are drawn. Suppose $G_{2}$ is 2-dimensional grid, which $k \geq 1, n_{1}, n_{2}$, is infinite. When $k$ is odd or even number, the $k$-distance chromatic number is $\frac{1}{2}(k+1)^{2}$ or $\frac{1}{2}\left((k+1)^{2}+1\right)$ respectively. Kim in[6] have studied the 2-distance coloring of the direct product of $m$ order path and $n$ order circle, who have proved that the number of 2-distance chromatic number equals to 5 or 6 .

Similarly $k$-distance edge coloring which the distance of any two edges is not more than $k$ need color differently. Due to the distance of edge coloring concept put forward later,Tian et al.in [7] studied the chromatic number of 2-dimensional grid $L_{2}$, get the precision value of chromatic number. When $k$ is odd, $\chi_{k}^{\prime}\left(L_{2}\right)=(k+1)^{2}$, when $k$ is even, the chromatic number is $k(k+2)$.

In this paper, we shall study the $k$-distance colorings and $k$-distance edge colorings of path double graphs, which is based on the semistrong product. Suppose $G$ is a simple connection graph, using $V(G)$ and $E(G)$ express vertex set and edge set of $G$ separately. And $(x)_{k}=x \bmod k$, the concepts of the double graphs and the semistrong product are as follows in definition 1.1 and 1.2, and all unknown symbols and terms in the text, see Ref [8] .

Definition 1.1 ${ }^{[8]}$ The semistrong product $G \bullet H$ has vertex set $V(G) \times V(H)$ and edge set $\left\{\left(u_{1}, v_{1}\right)\left(u_{2}, v_{2}\right) \mid u_{1} u_{2} \in E(G)\right.$ and $v_{1} v_{2} \in E(H)$ or $u_{1}=u_{2}$ and $\left.v_{1} v_{2} \in E(H)\right\}$. 
Definition 1.2 ${ }^{[9]}$ The double graph $D(G)$ of $G$ has a vertex set $V(G) \cup V(H)$ and an edge set $E(G) \bigcup E(H) \bigcup\left\{v_{i} v_{j}{ }^{\prime} \mid v_{i} \in V(G), v_{j}{ }^{\prime} \in V\left(G^{\prime}\right), v_{i} v_{j} \in E(G)\right\}$, graph $H$ copies graph $G$.Specifically, when $G=P_{n}$, we have $D(G) \cong P_{2} \bullet P_{n}$

The double graph of $n$ order path is isomorphic to the semistrong product graph of 2 order path and $n$ order path as shown in Figure 1.

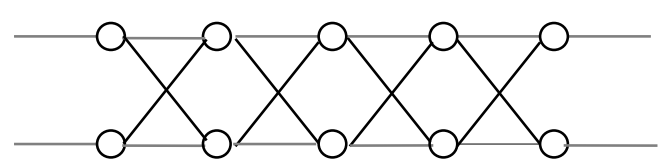

Figure $1 D\left(P_{n}\right) \cong P_{2} \bullet P_{n}$

Lemma 1.1 $H$ is an arbitrary subgraph of $G$, then $\chi_{2}(H)<\chi_{2}(G)$.

\section{Theorem and Proof}

Suppose $P_{n}$ is a path with vertex set $V\left(P_{n}\right)=\{0,1, \cdots . n-1\}$, and the vertices in $V\left(P_{n}\right)$ are adjacent if and only $|t-s|=1$. In order to get $k$-distance colorings of double path graph, we give the following lemma first.

Lemma 2.1 ${ }^{[8]}$ If the diameter of $n$ order simple graphs $G$ is not exceeding $k$, then $\chi_{k}(G)=n$.

The theorems and proofs about $k$-distance colorings and $k$-distance edge colorings of double graphs $D\left(P_{n}\right)$ are as follows.

Theorem 2.1 Let $P_{n}$ be a path of order $n, k$ is a positive integer greater than 2, then

$$
\chi_{k}\left(D\left(P_{n}\right)\right)=\left\{\begin{array}{l}
2(k+1), k \leq n-2, \\
2 n, k \geq n-1 .
\end{array}\right.
$$

Proof We know that $D\left(P_{n}\right)=P_{2} \bullet P_{n}$, and suppose $V\left(P_{2}\right)=\{0,1\}, V\left(P_{n}\right)=\{0,1, \cdots n-1\}$, then $V\left(D\left(P_{n}\right)\right)=\{(i, j) \mid i=0,1, j=0,1, \cdots n-1\}, G_{i}$ is export subgraph of $D\left(P_{n}\right), i=0,1$. Obviously every $G_{i}$ is a path of order $n$. There are two cases to be considered.

Case $1 k \leq n-2$.

For the distance of any two vertices $(i, j)$ and $(t, s)$ in the vertex set $B_{k}=\{(i, j) \mid i=0,1, j=0,1, \cdots k\}$ is no more than $k$, where $B_{k}$ is a subset of $V\left(D\left(P_{n}\right)\right)$ and $\left|B_{k}\right|=2(k+1)$,so we can obtain $\chi_{k}\left(D\left(P_{n}\right)\right) \geq 2(k+1)$. Now we prove $\chi_{k}\left(D\left(P_{n}\right)\right) \leq 2(k+1)$. For $D\left(P_{n}\right)$, we construct a $k$-distance vertex coloring using $2(k+1)$ colorings. First we use $(k+1)$ colorings color $G_{0}$, then using another $(k+1)$ colorings color $G_{1}$. Obviously this is $k$-distance vertex coloring of $D\left(P_{n}\right)$, $\chi_{k}\left(D\left(P_{n}\right)\right) \leq 2(k+1)$. From above we can obtain when $k \leq n-2, \chi_{k}\left(D\left(P_{n}\right)\right)=2(k+1)$.

Case $2 k \geq n-1$.

It can easily demonstrate the diameter of $D\left(P_{n}\right)$ is 2 , when $k \geq 2$, by lemma 2.1 , we have $\chi_{k}\left(D\left(P_{n}\right)\right)=2 n$. So the proof of Theorem 2.1 is already completed.

Theorem 2.2 The $k$-distance edge chromatic number of double graphs $D\left(P_{n}\right)$, we have

$$
\chi_{k}{ }^{\prime}\left(D\left(P_{n}\right)\right)= \begin{cases}4(n-1) & k \geq n-2 \\ 4(k+1) & k \leq n-3\end{cases}
$$

Proof When $k=2, n=2$, we know that $D\left(P_{2}\right) \cong C_{4}$, so $\chi_{2}{ }^{\prime}\left(D\left(P_{2}\right)\right)=4$.If $n=3$, the distance of every two edges of $D\left(P_{3}\right)$ is no more than 2, while $\left|E\left(D\left(P_{3}\right)\right)\right|=8$, so we can obtain the 2-distance chromatic number $\chi_{2}{ }^{\prime}\left(D\left(P_{3}\right)\right)=8$. Similarly, if $n=4, \chi_{2}{ }^{\prime}\left(D\left(P_{4}\right)\right)=\left|E\left(D\left(P_{4}\right)\right)\right|=12$. If $n \geq 5$, by 
lemma 1.1 we know $\chi_{2}{ }^{\prime}\left(D\left(P_{n}\right)\right) \geq 12$. Now we prove $\chi_{2}{ }^{\prime}\left(D\left(P_{n}\right)\right) \leq 12$. We construct a 2 -distance edge coloring, and let $\sigma$ be the 2-distance edge coloring using 12 colors. $\sigma((x, y)(x+1, y))=(x+9 y)_{12} ; \sigma\left((x, y)\left(x+1,(y+1)_{2}\right)\right)=\left(x+3(y)_{2}+1\right)_{12}$.

First we demonstrate $\sigma$ is a normal coloring. From the definition of $\sigma$, any vertex $(x, 1)$ or $(x, 0)$ in $D\left(P_{n}\right)$, all the possible correlation edges colorings are as follows.

$$
\begin{gathered}
\sigma((x, 1)(x+1,1))=(x+9)_{12} ; \sigma((x-1,1)(x, 1))=(x+8)_{12} ; \\
\sigma((x-1,0)(x, 1))=(x+2)_{12} ; \sigma((x, 1)(x+1,0))=(x+6)_{12} . \\
\sigma((x, 0)(x+1,0))=(x)_{12} ; \sigma((x, 0)(x+1,1))=(x+3)_{12} ; \\
\sigma((x-1,1)(x, 0))=(x+5)_{12} ; \sigma((x-1,0)(x, 0))=(x+11)_{12} .
\end{gathered}
$$

Obviously, the correlation edges of $(x, 1)$ and $(x, 0)$ color different. Hence $\sigma$ is a normal coloring of $D\left(P_{n}\right)$. Now we demonstrate $\sigma$ is a 2-distance edge coloring of $D\left(P_{n}\right)$. We take any two edges $e_{1}, e_{2}$, and $d\left(e_{1}, e_{2}\right)=d,(d=1,2)$. To prove $\sigma\left(e_{1}\right) \neq \sigma\left(e_{2}\right)$, there are two cases to be considered.

Case $1 d=1$.

Suppose $v=(x, y)$, where $x=0,1,2 \cdots n-1, y=0,1$. From the definition of $\sigma$, we know that the correlation edges of $(x, 1)$ and $(x, 0)$ color different colorings. Thus $d=1, \sigma\left(e_{1}\right) \neq \sigma\left(e_{2}\right)$.

Case $2 d=2$.

From the structure of $D\left(P_{n}\right)$, there are two subcases that $e_{1}$ follow.

Case 2.1 $e_{1}=(x, y)(x+1, y)$. The situations of $e_{2}$ are as follows.(It can be seen in figure 2.1).

$$
\begin{gathered}
e_{2} \in\left\{\left(x+\alpha_{1}, y\right)\left(x+\beta_{1}, y\right) \mid \alpha_{1}= \pm 2, \beta_{1}=-1,3\right\} \bigcup \\
\left\{\left(x+\alpha_{2},(y+1)_{2}\right)\left(x+\beta_{2},(y+1)_{2}\right) \mid \alpha_{2}=0, \pm 1, \pm 2, \beta_{2}=0, \pm 1,2,3\right\} \bigcup \\
\left\{\left(x+\alpha_{3}, y\right)\left(x+\beta_{3},(y+1)_{2}\right) \mid \alpha_{3}=-1, \pm 2, \beta_{3}=0,-1,3\right\} \bigcup \\
\left\{\left(x+\alpha_{4},(y+1)_{2}\right)\left(x+\beta_{4}, y\right) \mid \alpha_{4}=1, \pm 2, \beta_{4}=-1,2,3\right\} .
\end{gathered}
$$

From the definition of $\sigma$, we have $\sigma\left(e_{1}\right)=(x+9 y)_{12}, \sigma\left(e_{2}\right) \in\left\{\left(x+9 y+\alpha_{1}\right)_{12}\right.$, $\left.\left(x+9(y+1)_{2}+\alpha_{2}\right)_{12}, \quad\left(x+3(y)_{2}+1+\alpha_{3}\right)_{12},\left(x+3(y+1)_{2}+1+\alpha_{4}\right)_{12}\right\}$. If $\sigma\left(e_{1}\right)=\sigma\left(e_{2}\right)$, there exist $\alpha_{1}, \alpha_{2}, \alpha_{3}, \alpha_{4}$ to satisfy $\left(\alpha_{1}\right)_{12}=0,( \pm 9)_{12}=\left(\alpha_{2}\right)_{12},\left(9 y-3(y)_{2}\right)_{12}=\left(\alpha_{3}+3\right)_{12}$, or $\left(9 y-3(y+1)_{2}\right)_{12}=\left(\alpha_{4}+3\right)_{12}$. While $y=0$ or $y=1$, it conflict with the values of $\alpha_{1}, \alpha_{2}, \alpha_{3}, \alpha_{4}$. Thus $d=2$, when $e_{1}=(x, y)(x+1, y)$, we prove $\sigma\left(e_{1}\right) \neq \sigma\left(e_{2}\right)$.

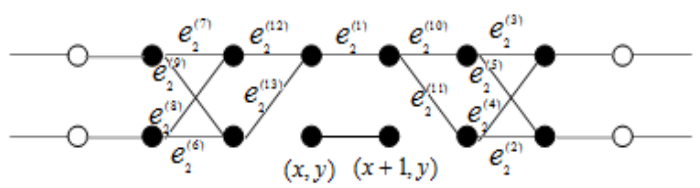

$2.1 e_{1}=(x, y)(x+1, y) \quad y=0$, cases of $e_{2}$, (black point connection)

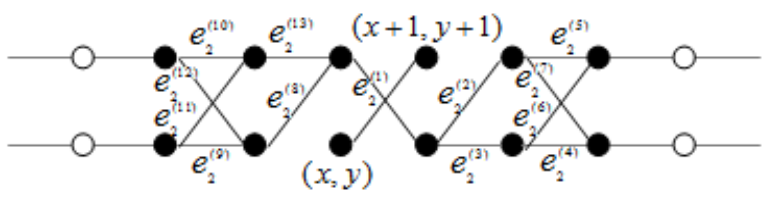

$2.2 e_{1}=(x, y)\left(x+1,(y+1)_{2}\right), y=0$, cases of $e_{2}$, (black point connection)

Fig. $2 d=2$ all possible adjacent edges of $e_{1}$

Case 2.2 $e_{1}=(x, y)\left(x+1,(y+1)_{2}\right)$. Kinds of $e_{2}$ cases can be seen in Figure 2.2. 


$$
\begin{gathered}
e_{2} \in\left\{\left(x+\alpha_{1}, y\right)\left(x+\beta_{1}, y\right) \mid \alpha_{1}=1, \pm 2, \beta_{1}=-1,2,3\right\} \bigcup \\
\left\{\left(x+\alpha_{2},(y+1)_{2}\right)\left(x+\beta_{2},(y+1)_{2}\right) \mid \alpha_{2}=-1, \pm 2, \beta_{2}=0,-1,3\right\} \bigcup \\
\left\{\left(x+\alpha_{3}, y\right)\left(x+\beta_{3},(y+1)_{2}\right) \mid \alpha_{3}= \pm 1, \pm 2, \beta_{3}=0,-1,2,3\right\} \bigcup \\
\left\{\left(x+\alpha_{4},(y+1)_{2}\right)\left(x+\beta_{4}, y\right) \mid \alpha_{4}=0, \pm 2, \beta_{4}=-1,1,3\right\} .
\end{gathered}
$$

From the definition of $\sigma, \sigma\left(e_{2}\right) \in\left\{\left(x+9 y+\alpha_{1}\right)_{12},\left(x+9(y+1)_{2}+\alpha_{2}\right)_{12},\left(x+3(y)_{2}+1+\alpha_{3}\right)_{12}\right.$, $\left.\left(x+3(y+1)_{2}+1+\alpha_{4}\right)_{12}\right\}$.

Suppose $\sigma\left(e_{1}\right)=\sigma\left(e_{2}^{(i)}\right), i=(1,2, \cdots 13)$, while $(\alpha)_{12}=0, \alpha \in( \pm 3,1,2,4,5,7,8,10,11)$, it shows contradictions in turn. Thus $e_{1}=(x, y)\left(x+1,(y+1)_{2}\right), \sigma\left(e_{1}\right) \neq \sigma\left(e_{2}\right)$. Hence, when $d=1,2$, $\sigma\left(e_{1}\right) \neq \sigma\left(e_{2}\right)$.

From case 1 and case 2 , we can conclude that $n \geq 5, \chi_{2}{ }^{\prime}\left(D\left(P_{n}\right)\right)=12$. Above all cases, when $k \geq n-2, \chi_{2}{ }^{\prime}\left(D\left(P_{n}\right)\right)=4(n-1)$, while $k \leq n-3$, the edge chromatic number equals $4(k+1)$.

Similarly, we can prove the $k$-distance edge chromatic numbers of $D\left(P_{n}\right)$, and have the same formula when $k \geq 3$.

The proof of Theorem 2.2 is completed.

\section{Acknowledgement}

This work was financially supported by Central University for Northwest Minzu University of the Basic Scientific Research business expenses of the Special Funds to support graduate projects (Yxm 2017105, Yxm2017103) and Innovative Team(Graph Theory and Intelligent Computing) Subsidize of Northwest Minzu University (120-112033) and Ethnic Affairs Commission of China (No.14XB Z018).

\section{References}

[1] Kramer F, Kramer H. Un problème de coloration des sommets d'un graphe[J]. Comptes Ren- dus de l'Académie des Sciences Paris Series A-B. 1969:A46-A48.

[2] Kramer F, Kramer H. Ein Farbungs problem der Knotenpunkte eines Graphen bezüglich der Distanz p[J]. Revue Roumaine de Mathema Tiques Pures et Appliquees. 1969, 14(2): 1031- 1038.

[3] Kramer F. Sur le nombre chromatique K(p,G) des graphes. RAIRO R-1. 1972: 67-70.

[4] Kramer F, Kramer H. A survey on the distance-colouring of graphs[J]. Discrete Mathematics. 2008, 308: 422-426.

[5] Fertin. G, Godard E, Raspaud. A. Acyclic and k-distance coloring of the grid[J]. Information Processing Letters. 2003, 87:51-58.

[6] Kim B M, Song B C, Rho Y. 2-distance colorings of some direct products of paths and cycles [J]. Discrete Mathematics. 2015, 338(10):1730-1739.

[7] Shuangliang Tian, Ping Chen. Distance Coloring and Distance Edge-Coloring of dimensional Lattice[C]. International Conference on Intelligent Computing Theories and Applications. 2012:1-7.

[8] Bondy J A . Graph Theory with Applications [M]. New York: American Elsevier. 1976.

[9] Jaradat M M M. On the edge coloring of graph products[J]. International Journal of Mathematics and Mathematical Sciences. 2005,16:2669-2676.

[10] Zhongfu Zhang et al. The double Graph and the Comlement Double Graph of a Graph[J]. Advanc- es in Mathematics. 2008, 37(3):303-310. 